\title{
Characterising Porosity in Platinum
}

\section{Nanoparticles}

\section{Wenmiao Yu' ${ }^{1}$, Christopher Batchelor-McAuley1*, Yi-Chi Wang ${ }^{2}$, Shou-Qi Shao' ${ }^{2}$, Simon M.}

Fairclough$^{2}$, Sarah J Haigh ${ }^{2}$, Neil P. Young ${ }^{*}$ and Richard G. Compton ${ }^{*}$

${ }^{1}$ Physical and Theoretical Chemistry Laboratory, Department of Chemistry, University of Oxford, South Parks Road, Oxford OX1 3QZ, United Kingdom

${ }^{2}$ School of Materials, University of Manchester, Oxford Road, Manchester M13 9PL, United Kingdom

${ }^{3}$ Department of Materials, University of Oxford, Parks Road, Oxford OX1 3PH, United Kingdom

* corresponding author emails:

Christopher.Batchelor-McAuley@chem.ox.ac.uk

Neil.Young@materials.ox.ac.uk

Richard.Compton@chem.ox.ac.uk

\begin{abstract}
Accurately determining the morphology and hence the true surface areas of catalytic nanoparticles remains challenging. For many chemically synthesised nanoparticle suspensions conventional BET surface area measurements are often not feasible due to the large quantities of material required. For platinum, a paradigmatic catalyst, this issue is further complicated by the propensity of this metal to form porous aggregate structures comprised of smaller (ca. 2-5 nm) crystallites as opposed to continuous solid structures. This dendritic/porous particulate morphology leads to a large but poorly defined 'active' surface which is difficult to measure accurately. Here we compare, single nanoparticle electrochemistry with three dimensional (3D) electron tomography and quantitative 2D high-angle annular dark-field (HAADF) scanning transmission electron microscopy (STEM) analysis to yield insights into the porosity and chemically accessible surface area of a $30 \mathrm{~nm}$ diameter commercial Pt nanoparticle catalyst. Good quantitative agreement is found between 2D and 3D STEM-based measurements of the particle morphology, density and size distribution. Both 3D STEM tomography and single nanoparticle electrochemical measurements allow quantification of the surface area but the electrocatalytic surface area is found to be $2.8 \mathrm{X}$ larger than is measured in STEM; indicating the importance of the atomic scale roughness and structure $(<2 \mathrm{~nm})$ in contributing to the total catalytic surface area of the nanomaterial.
\end{abstract}

\section{KEYWORDS}

Porosity; Electrochemistry; Electron Tomography; HAADF-STEM, Platinum Nanoparticles 


\section{Introduction}

Aggregation based growth is a fundamentally important pathway in the chemical synthesis of platinum nanomaterials. ${ }^{1-3}$ However, the resulting larger platinum nanoparticle structures are porous or dendritic in morphology. ${ }^{4}$ This is in stark contrast to silver and gold nanomaterials which tend to form highly dense solid structure ${ }^{5}{ }^{6}$ and may be chemically produced in sizes that range from nanometers to micrometers. ${ }^{7,} 8$ Larger solid platinum particles can be generated electrochemically ${ }^{9}$ or via laser ablation ${ }^{10}$ but even when larger solid platinum structures have been formed via other physical techniques, such as ion-beam deposition, the resulting material may still be found to be formed of much smaller distinct crystalline parts. ${ }^{11}$ These composite aggregate or dendritic platinum structures have been used extensively as active catalysts; being employed for instance in both nanomotors and as potential nanomedicines. ${ }^{12-16}$ However, to optimise the properties of such materials it is important to answer the question, 'what is the morphology and hence accessible surface area of these aggregates?'.

For bulk nanoporous materials, BET analysis ${ }^{17}$ is the de facto standard for determining surface areas but it is not readily applicable for the analysis of many nanomaterials. First, BET needs relatively large quantities of material, often a total sample surface area of $>0.1 \mathrm{~m}^{2}$ is needed for analysis. ${ }^{18}$ For metallic nanoparticles this practically implies the need to use at least $0.1-0.01 \mathrm{~g}$ of material (depending on the material composition and structure); this may be both challenging and expensive to synthesize. Secondly, the sample must be clean and dry, while many chemically synthesised nanoparticles are prepared in the solution phase, in the presence of surfactants (capping agents) and at comparatively low concentrations $c a . \mathrm{mg} / \mathrm{mL}$. Third, the BET technique, as stressed in the ISO international standard, ${ }^{19}$ is only applicable to type II and IV isotherms. The presence of micropores $(<2 \mathrm{~nm})$ will lead to a type I isotherm and, as will be evidenced in this work, the platinum nanoparticles studied herein contain smaller micropore structures, thus rendering BET analysis unsuitable for this material. Nevertheless, accurate surface area measurements are key to understanding the chemical catalytic activity of nanomaterials. For a given interfacial reaction assuming that the process is not mass-transport limited - then the reaction rate will be a function of the number and inherent activity of the surface sites. To a first approximation the number of active sites is proportional to the total surface area. Consequently, if we wish to understand the chemical activity of the material then the measured reaction rate needs to be normalised against the true interfacial surface area.

The surface area of platinum may be determined electrochemically using a variety of surface specific redox processes, such as for example, hydrogen underpotential deposition or removal, carbon 
monoxide oxidative stripping or direct oxidation of the platinum surface. By measuring the charge passed during such oxidation and reduction processes and taking an assumed ratio between the charge and surface area then the so-called electrochemical surface area (ECSA) can be measured. This type of measurement is routinely made on large ensembles of nanoparticles and underpins a significant proportion of the work in the field of materials electrocatalysis. ${ }^{20}$ First, determining the average per particle surface area requires an estimate of the nanoparticle number density; this necessitates the use of an independent measurement technique. Moreover, these large ensemble measurements are unable to give insight into the heterogeneity of a nanoparticle sample. Second, this issue of the total number of nanoparticles being measured in the ensemble electrochemical experiment is further complicated by possible variability in the nanoparticle/electrode connectivity. This problem of electrical nanoparticle connectivity results in a systematic error which necessarily leads to some level of underestimation of the true nanoparticle surface area. Experimentally, even in the best case scenarios, it is commonly encountered that the measured electrochemical surface area is at least a third less than anticipated based on the quantity of used material. ${ }^{21}$ This discrepancy can, at least in part, be rationalised on the basis of the substrate blocking the catalytic (platinum) interface but clearly other sources of uncertainty will contribute to this error.

As an alternative, a recently developed single nanoparticle electrochemistry technique has enabled individual platinum nanoparticle surface areas to be measured. ${ }^{22}$ Here a microelectrode is immersed into a solution containing a suspension of platinum nanoparticles and hydrogen gas. The hydrogen gas dissociatively chemisorbs on to the platinum surface and if the nanoparticle collides with an electrode potentiostated to a suitably oxidising potential then the chemisorbed layer of hydrogen atoms can be electrochemically oxidatively removed from the individual particle surface. The charge associated with this nano-event gives a quantitative measure of the total number of electrons passed during the course of the reaction and hence the total surface area of the nanostructure.

Transmission electron microscopy (TEM) provides a complementary method of surface area measurement for individual nanoparticles. For particles with a known geometry, the surface area can be estimated based on the 2D projected perimeter imaged in the TEM. For more complicated structures the 3D morphology can be estimated from a 2D image by careful image interpretation. The high-angle annular dark field scanning transmission electron microscopy (HAADF-STEM) imaging mode provides 'Z-contrast' images; a form of mass thickness contrast that yields more readily physically interpretable images than conventional TEM data. For a single element specimen, the HAADF-STEM image intensity varies monotonically as a function of the material's thickness. Consequently, for simple monometallic or bimetallic systems quantitative analysis of the variation in 
intensity across a single 2D HAADF-STEM image can allow the projected thickness, volume and composition of a nanomaterial to be assessed. This approach has been successfully applied to a number of systems including the analysis of core shell and multilayer particles, ${ }^{23,24}$ and semiconductor dopant distributions. ${ }^{25}$ However, for porous nanostructures or more complex larger nanoparticles a more accurate description of the 3D structure, morphology and elemental distribution can be recovered using electron tomography. ${ }^{26-28}$ The electron tomography technique is based upon acquiring a tilt series data set with different specimen tilts (typically $\pm 70^{\circ}$ ) and using these different projections to reconstruct the $3 \mathrm{D}$ dimensional structure and morphology of the material.

In this work, we demonstrate the porous structure and internal heterogeneity of platinum nanoparticles through the combination of electron tomography, quantitative HAADF-STEM analysis and single nanoparticle electrochemistry. Solid laser ablated platinum nanoparticles provide a calibration standard against which the internal density of the mesoporous structure can be analysed, evidencing a more general method of the quantitative analysis of larger mesoporous structures in HAADF-STEM. From this work the contribution of the molecular scale and structure of the nanoparticle towards its surface area is evidenced; micropores $(<2 \mathrm{~nm})$ in the internal structure of the material are both present and chemically accessible. Discrepancies in the measured surface area of the material between different techniques relate to the length scale and resolution at which the measurement is being made. We show that combining the three techniques is a powerful approach, with the electrochemical measurements providing insight into the catalytically active surface area while the HAADF-STEM (tomography and image analysis) provides morphological information and allows estimation of the size dispersion and volume of metal in each nanoparticle.

\section{Experimental}

Chemicals

Hydrogen $\left(\geq 99.98 \% \mathrm{H}_{2}\right)$ was supplied from BOC, Surrey, U.K. Sodium nitrate $\left(\geq 99.0 \%, \mathrm{NaNO}_{3}\right)$ and sodium hydroxide $(\geqslant 99.3, \mathrm{NaOH})$ were obtained from Sigma-Aldrich, Dorset, U.K and Fisher Chemicals, NH, USA respectively. Citrate-capped porous platinum nanoparticles (porous PtNPs) were obtained from nanoComposix, San Diego, CA, with a nominal diameter of $30 \mathrm{~nm}$ (characterisation provided in text) and concentration of $0.05 \mathrm{mg} / \mathrm{mL}$. Solid laser ablated platinum nanoparticles (solid PtNPs) were provided by Particular GmbH, Burgdorf, Germany, with a reported diameter of $50 \mathrm{~nm}$ and concentration $100 \mathrm{mg} \mathrm{L}^{-1}$. All solutions were prepared with ultrapure water from Millipore, with a resistivity of $18.2 \mathrm{M} \Omega \mathrm{cm}$ at $298 \mathrm{~K}$. 
Single Nanoparticle Electrochemistry Experiments

All nanoimpact electrochemical experiments were performed on a three electrode system in a Faraday cage. The working electrode was a gold microdisc electrode of diameter $10.0 \mu \mathrm{m}$ from ALS, Tokyo, Japan and was polished with alumina powders of decreasing sizes: 1.0, 0.3 , and $0.05 \mu \mathrm{m}$ from Buehler, Lake Bluff, IL. The reference electrode was a mercury-mercurous sulfate electrode from BASi, West Lafayette, IN. A platinum foil from Goodfellow, Cambridge, U.K., acted as the counter electrode. All experiments were conducted under a hydrogen atmosphere and all electrochemical measurements were thermostated at $25 \pm 0.5^{\circ} \mathrm{C}$. Potential and impact current measurements were controlled by a lab built highly stabilised potentiostat. An NI USB-6289 data acquisition (DAQ) device from National Instruments, Austin, TX, was connected to a computer interface via a USB. Python 3.5 (Canopy from Enthought, Austin, TX) was employed to control the DAQ. The working electrode was running to ground where a low-noise current amplifier Axopatch 200b, Molecular Devices, SD, USA, was used to amplify the current and filtered at an analogue frequency of $10 \mathrm{kHz}$. The DAQ device oversampled and digitized the incoming analogue signal at a stream rate of $625 \mathrm{kHz}$, and the digitized signal was subsequently filtered at a bandwidth of $250 \mathrm{~Hz}$. The filter and measurement systems used in this work conserve the charge passed.

\section{Transmission Electron Microscopy}

Samples were prepared by depositing a drop of the stock mesoporous or solid PtNP aqueous suspension, with concentration $100 \mathrm{mg} \mathrm{L}^{-1}$ onto a 200 mesh carbon grid coated with holey carbon film from Agar Scientific, Stansted, U.K. To reduce contamination TEM grids were stored in vacuum overnight prior to imaging. TEM and HAADF STEM imaging was performed using a JEOL JEM-3000F FEGTEM operated at $300 \mathrm{kV}$ with a convergence angle of $25 \mathrm{mrad}$ and a HAADF inner angle of 55 mrad. Electron tomography was performed using a FEI G2 probe-corrected X-FEG Titan with an accelerating voltage of $200 \mathrm{kV}$ a convergence angle of $21 \mathrm{mrad}$, a HAADF inner angle of mrad 64 mrad. The tilt series data set was acquired with an angular range of -72 to +78 degrees at 2 degree increments. ImageJ software ${ }^{29}$ was used to perform 2D size analysis and total variation minimisation reconstruction was performed in the TomViz software. ${ }^{30}$

\section{Results and Discussion}

In this work we study the structure of a commercially available, chemically synthesised platinum nanostructure; examples of similar materials are found through-out the literature. ${ }^{1,4,12,13}$ These nanoparticles are porous/dendritic in structure and are comprised of an aggregate of smaller crystalline structures. This work compares surface area and volume measurements from HAADF 
STEM electron tomography, HAADF STEM quantitative 2D analysis and electrochemical single nanoparticle measurements. Electron tomography provides a 3D model, yielding both the volume of metal relative to the 2D 'effective' diameter of the particle and the structure's metallic surface area. However the tomography approach is too time consuming to apply to many nanoparticles, requires a high electron dose and data interpretation requires thresholding which can be subjective. By comparison, quantitative analysis of the 2D HAADF-STEM images provides a less time consuming method to obtain the average density of metal in the particle. High density spherical platinum nanoparticles produced by laser ablation are used to provide an internal standard for the 2D STEM measurements, vide infra. These particle densities as measured from the 2D image are subsequently directly compared to the electron tomography analysis. Finally, electrochemical single nanoparticle measurements provide an independent verification of the direct surface area of individual nanoparticles in solution.

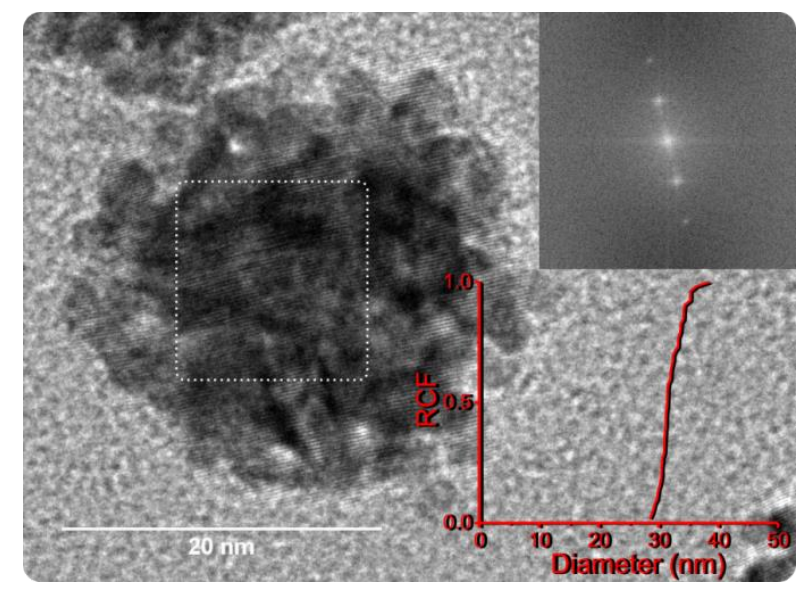

Figure 1 Representative HR-TEM image of the porous platinum nanoparticles, highlighting the composite aggregate structure of the material and the crystallinity of the platinum. The fringing spacing and direction is evidenced in the FT of the image of the particle (top right inlay, with FT taken from the area highlighted by the dotted white box). Also inlayed is a relative cumulative frequency distribution (RCF) for the overall aggregate size giving an average particle diameter of $31 \pm 2 \mathrm{~nm}(\mathrm{n}=47)$.

Figure 1 depicts a representative HR-TEM image of a mesoporous platinum nanoparticle with the measured relative cumulative frequency size distribution (sample size, $n=47$ ) for the nanoparticle population inset bottom right. Note that in this work we use the relative cumulative frequency (i.e. the cumulative frequency divided by the sample size, see SI section 1 for more details) to visualise the nanoparticle size distribution. This choice arises simply from the fact that how data is presented in a histogram size distribution is, to an extent, subject to the used 'bin size'. The histogram is directly related to the derivative of the cumulative frequency plot. For the inlay of figure 1 the 'effective' nanoparticle diameter (d) has been determined from measurement of the projected area of the nanoparticle (A) in the $2 \mathrm{D}$ image, as $d=\operatorname{sqrt}(4 \mathrm{~A} / \mathrm{pi}$ ) (for details see $\mathrm{SI}$ Section 1 ). The nanoparticulate material was determined to have an average diameter of $31 \pm 2 \mathrm{~nm}$ but the HR-TEM 
images (Figure 1) clearly showed that each particle consisted of an aggregation of many smaller crystallites. The lattice fringes observable in the HR-TEM images and their Fourier transforms (FTs) (example inlayed top right Figure 1) showed that the component aggregated crystallites are largely aligned to a single crystallographic zone axis, suggesting that the particles attach via oriented attachment. ${ }^{1}$ On the edge of the particle, where fewer crystallites are overlapping it is possible to measure their diameters from the HR-TEM images as $3 \pm 0.5 \mathrm{~nm}$ (sample size, $\mathrm{n}=15$ ). However, the mesoporous internal structure of the particle is too complex to interpret from a single HR-TEM image. This paper serves to provide insight into the internal structure and morphology of the particulate material evidencing its porosity and demonstrating that, despite the particle's narrow size distribution, the individual particles have a large heterogeneity in their internal porosity.

\section{Electron Tomography}

In order to visualise the internal structure of the platinum nanomaterial a tilt series containing $752 \mathrm{D}$ HAADF-STEM projections of a particle was recorded across angles ranging from -72 to 76 degrees with 2 degree increments. From this tilt series a tomogram was reconstructed using a total variation minimisation method; ${ }^{31}$ further details on the imaging and reconstruction method can be found in previous papers $^{32}$ and the SI Section 2 along with additional examples of other imaged and reconstructed particles. Figure 2 presents the reconstructed data for a platinum nanoparticle, the top left image presents an iso-surface 3D model of the particle as viewed down the $Z$ axis. The remaining three panels provide cross-sectional views through the centre of the nanoparticle in the $\mathrm{XY}, \mathrm{ZY}$ and $\mathrm{XZ}$ planes.
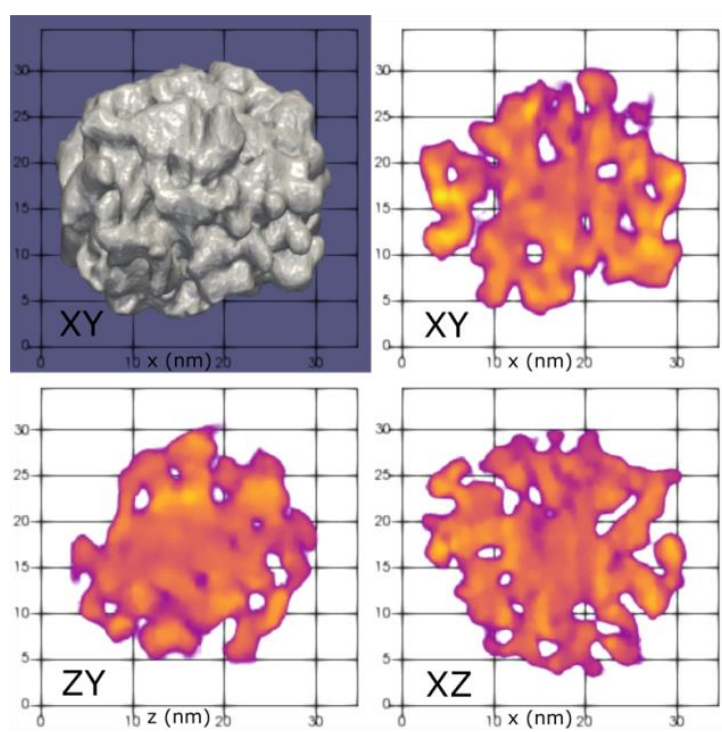

Figure 2: Tomogram reconstruction of a nominally $30 \mathrm{~nm}$ platinum nanoparticle. Top left: rendered view of the 3D reconstruction viewed down the z-axis. Top right, bottom left and bottom right represent perpendicular cross-sectional views through the nanoparticle in the $X Y, Z Y$ and $X Z$ planes respectively. 
The data presented in Figure 2 clearly evidences the mesoporous structure of the particle and the presence of nanometer sized internal pores. From this three dimensional structure both the total volume of the metal and its associated surface area can be estimated. A total of five particles have been reconstructed in this manner and this allows the volume of the metal in an average individual nanoparticle is estimated as being $8,100 \pm 900 \mathrm{~nm}^{3}$ with a corresponding surface area of $4,600 \pm 800$ $\mathrm{nm}^{2}$ (errors represent standard deviation for five measurements). Furthermore, from the tomographic data it is possible to estimate the density of the nanoparticle. Here this is done considering an effective volume based on the average 'effective' diameter of the particles (determined from the average of five images of each particle at different angles in the tilt series). From this measurement of the effective particle diameter for the five tomographically reconstructed particles, accounting for both the platinum metal and pores/voids in the material, this measurement gives an average inscribed volume of a nanoparticle as $11,600 \pm 1100 \mathrm{~nm}^{3}$. Consequently, on the basis of these tomography measurements the platinum metal is calculated to fill ca. $70 \pm 5 \%$ of the internal nanoparticle structure, hence ca. $30 \pm 5 \%$ of the nanoparticulate structure is comprised of pores in the material.

In reporting the above values it is important to reflect on the resolution of the used HAADF-STEM tomography method. Tomographic data was acquired with a STEM probe size at the entrance surface of the specimen of $\sim 0.25 \mathrm{~nm}$. However, by the exit surface of the nanoparticle the electron probe is estimated to have broadened by $\sim 1.3 \mathrm{~nm}$ due to the size of the nanoparticles $(30 \mathrm{~nm})$ and the $21 \mathrm{mrad}$ beam convergence angle. This beam broadening will decrease the achievable voxel resolution for this tomographic imaging in the centre of the particle as 1-2 $\mathrm{nm}$, although atomic resolution is readily achievable for individual (S)TEM images of the particle edges. Consequently, although the HAADF-STEM tomogram can successfully evidence the presence of larger mesopores $(>2 \mathrm{~nm})$ in the internal structure of the material it may not identify the smallest internal micropores $(<2 \mathrm{~nm})$. Further discussion of the accuracy of electron tomographic analysis, including the effect of the choice of thresholding intensity are provided in the SI Section 2.

\section{Quantitative HAADF-STEM imaging}

It is prohibitively time consuming to acquire tilt series tomographic data at this resolution for a statistically significant number of nanoparticles. Therefore it is desirable to obtain the same volume and surface area information via more scalable characterisation routes. Tomographic reconstruction algorithms rely on the intensity of the HAADF-STEM input images being a monotonic function of the density of the material. The HAADF-STEM image intensity is proportional to the fraction of the electron probe that has been scattered to high angles and consequently, the density of the material 
can be gained from a single HAADF STEM image given a suitable standard. This is a much faster approach for obtaining the mass of the particle as compared to electron tomography and has been shown to be highly successful for measuring the mass of size selected small metallic clusters $(<5 \mathrm{~nm}$ diameter), from quantitative atomic scale imaging. ${ }^{33}$ For ultra-small particles, individual atoms provide a useful calibration reference ${ }^{33,34}$ but for the larger nanostructures used in this work an alternate standard is required. Highly dense spherical platinum nanoparticles, as synthesised by laser ablation, provide the required calibration standard (Figure 3). ${ }^{35}$

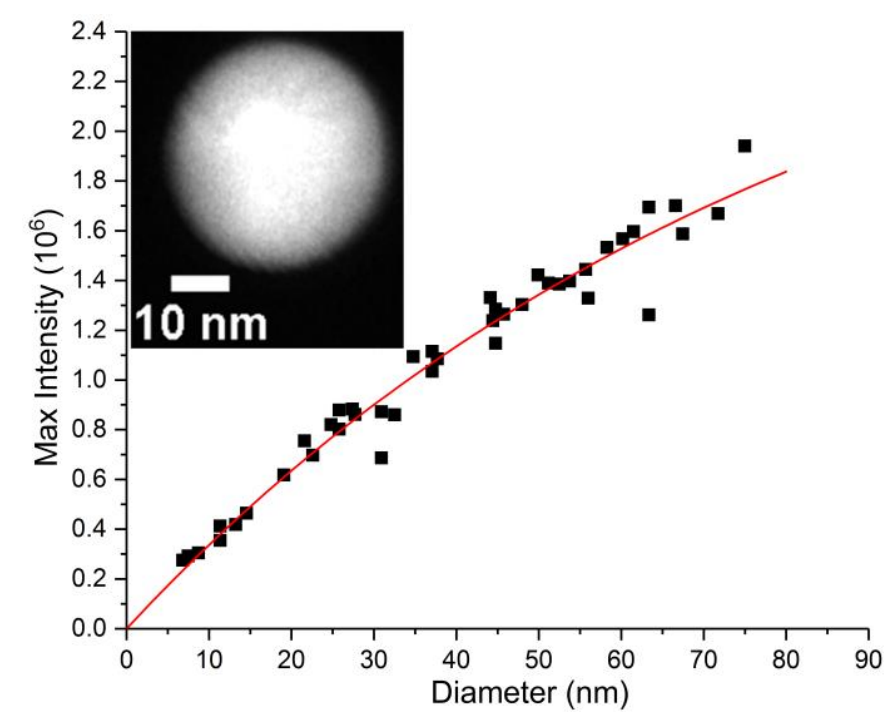

Figure 3 The measured maximum HAADF-STEM image intensity (arbitrary units) for a series of solid spherical laser ablated nanoparticles plotted against their measured diameter. The red line represents the best fit of the function $I_{\max }(1-\exp (-\mu D)),\left(R^{2}=0.963\right)$. Inlayed is an example HAADF-STEM image of a spherical laser ablated particle.

The inlay of Figure 3 demonstrates that the laser ablated platinum nanoparticles are highly regular and spherical (further HR-TEM images of these particles are provided in the SI section 3, demonstrating their high level of crystallinity). The laser ablated nanoparticles span a wide size range, with an average diameter of $39 \mathrm{~nm}$ and an associated inter-quartile size range of 25-56 nm. In many applications such a wide size distribution is problematic but in the present case it renders the material ideal for use as an internal standard. Figure 3 plots the maximum background corrected HAADF-STEM image intensity for a number of laser ablated nanoparticles (sample size, $n=43$ ) against the measured particle diameter. A small minority of the laser ablated particles were excluded from the data because they were observed to be ellipsoidal rather than spherical in shape, or because they showed significant asymmetry or internal structure (indicated by some amount of diffraction contrast) which could affect the attenuation of the electron beam.

Figure 3 shows that the laser ablated particle's maximum HAADF-STEM image intensity increases monotonically over the full range of particle sizes $(5-80 \mathrm{~nm})$. This maximum value of the image intensity occurs at the centre of the image of the particle where the platinum material is thickest. 
The SI Section 4 gives further details on how this maximum intensity is determined and the required background correction. The relationship between the particle diameter and the image intensity is not linear due to the attenuation of the electron beam intensity as it passes through the imaged material (cf. Beer-Lambert law). ${ }^{36}$ Consequently, this decrease in scattering intensity (and the resulting deviation from linear behaviour) can be suitably parameterised using the expression:

$I=I_{\max }(1-\exp (-\mu t))$

where I is the image intensity, $I_{\max }$ is the maximum image intensity (in the absence of the detector saturation), $\mu$ is the attenuation coefficient $\left(\mathrm{nm}^{-1}\right)$ and $\mathrm{t}$ is the sample thickness $(\mathrm{nm})$. The sample thickness, $t$, here refers to the thickness of platinum through which the electron beam has passed during the course of the imaging process. From fitting of equation 1 to the above experimental data the attenuation coefficient for this experimental setup is found to be $1.2 \pm 0.2 \times 10^{-2} \mathrm{~nm}^{-1}$. This measured attenuation coefficient is in good agreement with other measurements provided in the literature for comparable experimental setups. ${ }^{36,37}$ The attenuation coefficient is equal to the inverse of the mean free path beam electrons between scattering events and gives an indication of the non-linearity of the image intensity as a function of the material thickness. Importantly, the results present in Figure 3 clearly evidence that the image intensity of the HAADF-STEM image varies monotonically as a function of the thickness of the materials, such that the image intensity gives a route by which the amount of material per particle can be quantitatively analysed.

In the following analysis we do not simply measure the maximum image intensity, as done in Figure 3 , but integrate the image intensity across the entire 2D projected image of the nanoparticle. In this way we gain a measurement of the average mass-thickness of the particle. The relationship between integrated HAADF image intensity and nanoparticle mass can be applied to measure the mass of the chemically active porous platinum nanoparticles that are the focus of this study. A TEM grid was produced with both porous and solid platinum nanoparticles, so that the solid laser ablated nanoparticles act as an internal reference against which the image intensity of the porous nanoparticles could be calibrated.

Figure 4 a) shows a HAADF-STEM image of several aggregated platinum nanoparticles. The integrated HAADF image intensity for each porous nanoparticle was calculated for each particle. Figure $4 \mathrm{~b}$ ) depicts the measured integrated HAADF-STEM image intensity of the porous platinum nanoparticles divided by (normalised to) the image intensity expected for a solid platinum nanoparticle of the same diameter. The raw data showing the integrated nanoparticle intensity as a function of the particle diameter is depicted in the SI section 5 . On the normalised scale shown in 
Figure $4 \mathrm{~b}$ ) if the porous particle had the same scattering intensity as that of a solid particle it would have a value of 1.0. As expected all the porous particles are found to have a lower integrated intensity than the dense laser ablated particles with an average intensity of $0.7 \pm 0.09$. This relative intensity gives a direct estimate of the density of each nanoparticle metallic density estimated as $70 \pm 9 \%$ which is in good agreement with the electron tomography results of $70 \pm 5 \%$. The data shown in Figure $4 \mathrm{~b}$ ) indicates that there is no clear correlation between nanoparticle diameter and density. Moreover, the overall nanoparticle density varies quite considerably for nanoparticles with similar diameters, with fill factors varying from 54 to $96 \%$. These results indicate that although the nanoparticles are comparatively uniform in diameter and geometry, variations in internal structure lead to large heterogeneities in density and hence internal surface area.
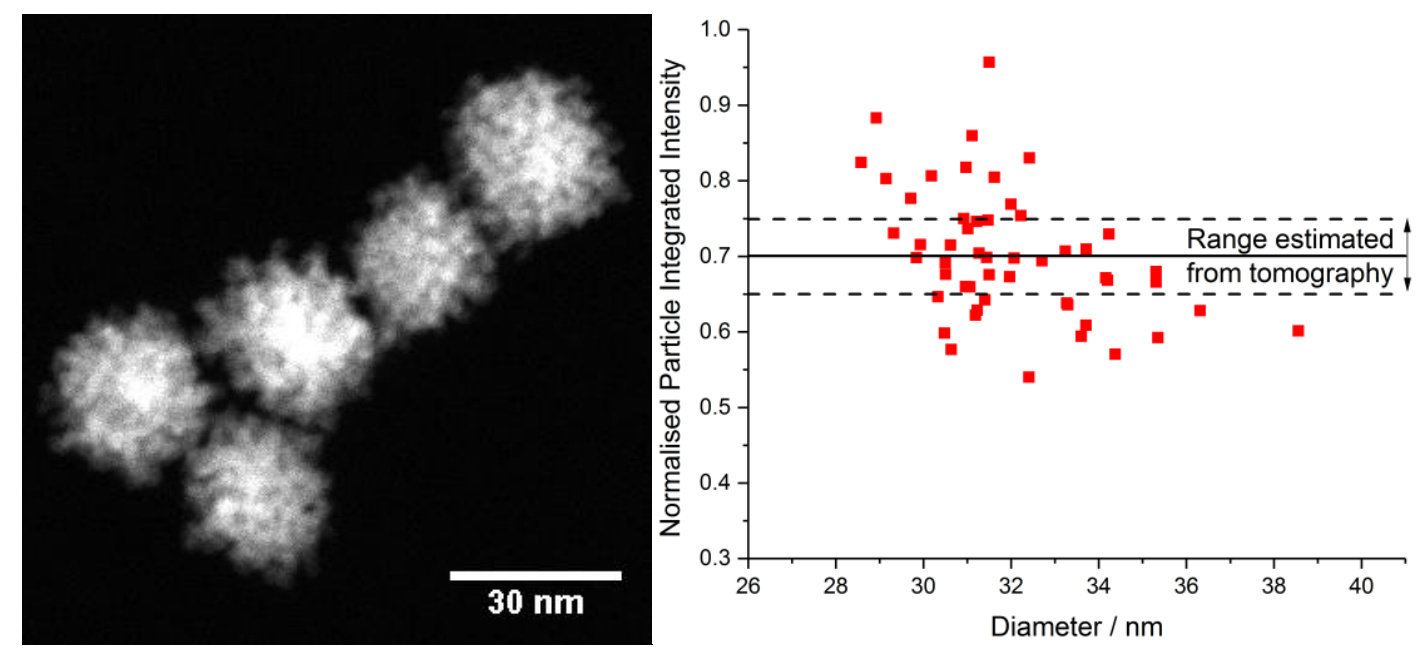

Figure 4 A) HAADF-STEM image of some representative porous platinum nanoparticles. B) The measured HAADF-STEM integrated image intensity for the porous platinum nanoparticles relative to that expected for a solid laser ablated particle of the same diameter. Horizontal line indicates the average value of $0.7 \pm 0.09$. This relative image intensity is taken as a measure of the particles overall metallic density consequently, the horizontal lines represent with porous particle density of $0.7 \pm 0.05$ as measured by the HAADF-STEM electron tomography.

Although the overall density of the particles as determined by the HAADF-STEM image analysis and electron tomography are in good agreement (as evidenced in Figure 4) it is worthwhile further considering the accuracy and limitations of this proposed image analysis procedure. To this end SI section 6 provides a simulation study evidencing the accuracy of using the 2D HAADF-STEM integrated image intensity to provide a measure of the overall density of a particle. The conclusion of which is that this method potentially underestimates the density of the particle by up to $3-5 \%$. This underestimation arises from the difficulty in determining the appropriate 'effective' diameter of the porous (highly non-spherical) platinum nanoparticles. This issue of defining the effective diameter of the particle is further compounded by the $2 \mathrm{D}$ resolution of the microscope images. The method of defining the nanoparticles effective diameter relative to its $2 \mathrm{D}$ projected area was adopted in part due to its ease of use. It should be further commented that in this image analysis the 
effect of the non-linearity of the scattering intensity of the material, as experimentally evidenced in Figure 3 has not been directly accounted for in this analysis procedure. First, due to the intensity being integrated over the whole particle the effect of this beam attenuation will be less, moreover we restrict ourselves to only analysing relatively small nanoparticles $(<40 \mathrm{~nm})$. For a particle $40 \mathrm{~nm}$ in diameter the maximum image intensity will be $21 \%$ less than would have been measured for a particle had the beam not been attenuated through the structure, whereas the integrated 2D image intensity is only reduced by $14 \%$. The difference between these two values reflects the fact that the particle thickness in the z-direction is not uniform - the particles are spherical. Second, in this analysis only particles of a similar size are compared. Over a small range of sizes the particle intensity will vary approximately linearly facilitating a direct comparison between the porous and solid nanoparticles. If larger $(>40 \mathrm{~nm})$ porous particle were to be analysed by this method a more sophisticated approach, enabling the non-linearity of the image intensity to be more rigorously accounted for, would likely need to be adopted.

\section{Single Particle Electrochemical Surface Area Measurements}

For platinum the molecular or ' $r e a l^{38}$ surface area of individual nanomaterials is experimentally accessible via single particle 'nano-impact' electrochemical analysis. Conventionally the charge associated with the addition or removal of a monolayer of adsorbed material is used to estimate the Electrochemical Surface Area (ECSA), ${ }^{20}$ common examples include the underpotential deposition or removal of hydrogen, the stripping of adsorbed carbon monoxide or the oxidation of the surface metal to its oxide. ${ }^{38,39}$ Figure 5 shows a representative experimental spike in current associated with the electrochemical removal of a chemisorbed hydrogen layer from an individual porous nanoparticle during an individual nano oxidative event. The chemisorbed layer of hydrogen is formed due to the presence of dissolved hydrogen gas in the solution phase; adsorption and homolytic cleavage of the gas leads to the modification of the nanoparticle surface. Integration of the spike in current associated with the arrival of an individual nano entity yields a measure of the total charge passed. This charged associated with the single nano-event can subsequently be used to yield an estimate of the surface area of the impacting nanomaterial. This estimation is achieved through the use of Faraday's first law and requires the assumption that the hydrogen initially forms a full monolayer on the platinum nanoparticle surface and that complete removal of this monolayer is achieved during the course of the oxidation event. Further details regarding this measurement technique and its potential limitations are covered in the SI section 7. Using a charge density ${ }^{38}$ of 2.1 $\mathrm{C} \mathrm{m}^{-2}$, the charges associated with 42 individual nano redox events have been converted into an estimated platinum surface area. The resulting surface area distribution is depicted in the inlay of 
Figure 5 as a relative cumulative frequency distribution. The average particle surface area as measured by the single nanoparticle electrochemical technique is found to be $13000 \pm 3100 \mathrm{~nm}^{2}$.

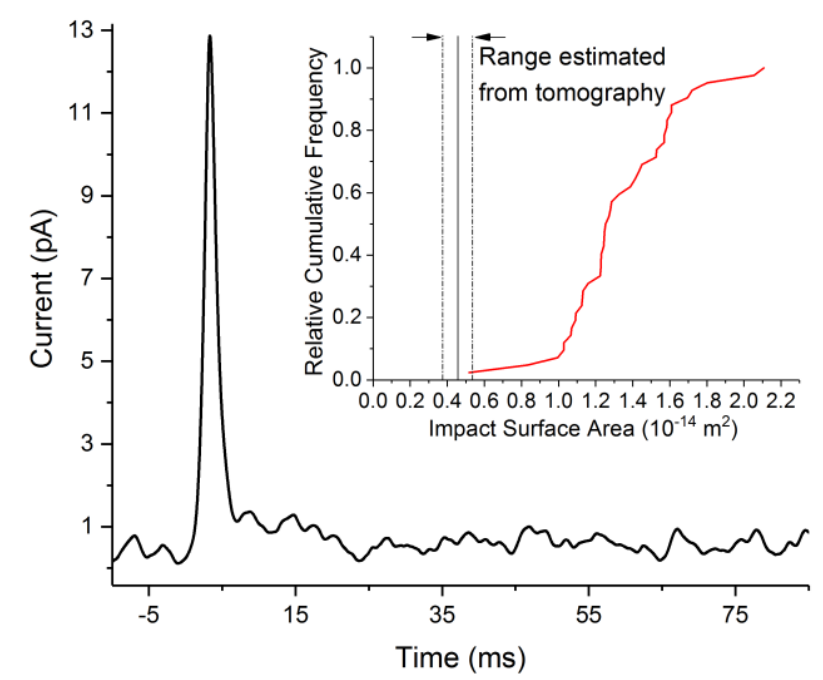

Figure $5 \mathrm{~A}$ representative example of an oxidative spike in current associated with the removal of chemisorbed hydrogen from an individual porous platinum nanoparticle. Assuming a conversion of $2.1 \mathrm{C} \mathrm{m}^{-2}$, the inlay depicts nanoparticle surface area distribution showing the normalised cumulative frequency plot of the estimated platinum nanoparticle surface areas. The electrochemically measured platinum nanoparticle surface area is found to have an average of $13000 \pm 3100 \mathrm{~nm}^{2}$, also depicted on the inlay is the nanoparticle surface area estimated from HAADF-STEM tomography.

Figure 5 shows a relatively large standard deviation for the electrochemically measured surface area, which in-part reflects the non-uniformity of the nanoparticle sample. This indicates that although the overall aggregate particle size distribution is relatively narrow (diameter $31 \pm 2 \mathrm{~nm}$, see Figure 1) the total nanoparticle surface area varies by over a factor of two across the nanoparticle distribution. This relatively large surface area distribution is in accordance with the wide range of particle densities as evidenced by the quantitative HAADF-STEM analysis (see Figure 4). However, the electrochemically measured surface area is $2.8 \mathrm{X}$ larger than the surface area of $4600 \pm 750 \mathrm{~nm}^{2}$ estimated from the HAADF-STEM tomography (shown by the vertical line in Figure 5 inset). The 3D STEM tomography surface area is outside even the tail of the electrochemical surface area suggesting the difference is not simply due to the small tomographic sample size. Furthermore, the 3D STEM tomography data is a close fit to the mean 2D STEM data (Figure 4), suggesting that the tomography data is representative of the population as a whole. Notably direct comparison of the single nanoparticle electrochemical data to that of the HAADF-STEM 2D image analysis is not feasible. In this experimental case the electrochemistry provides a measure of the particle surface area whereas the image analysis yields a measure of the volume of metal per particle. These two values can only be compared if assumptions regarding the structure and morphology of the nanoparticulate material are made. 
To understand the difference in the measured values of surface area for electrochemical measurements compared to HAADF STEM tomography it is therefore important to consider the different length scales of the two measurements. First however, we consider what magnitude of the single nanoparticle surface area we may anticipate for such a sample. A perfect sphere $31 \mathrm{~nm}$ in radius has an associated surface area of $3,019 \mathrm{~nm}^{2}$, relatedly a sphere with a rough surface, with a roughness on the length scale of $3 \mathrm{~nm}$ (i.e. comparable to the size of the substituent crystallites), would be anticipated to have a surface area of the order of $5,000 \mathrm{~nm}^{2}$. Alternatively, if we have an aggregate structure $31 \mathrm{~nm}$ in diameter and comprised of $3 \mathrm{~nm}$ substituent particles (with a fill factor of 0.7$)$ where all of the particle's surface is accessible to the solution phase, then the aggregate particle would be expected to have a total surface area of $\sim 22,000 \mathrm{~nm}^{2}$. Both the tomographically measured $\left(4,600 \mathrm{~nm}^{2}\right)$ and electrochemically determined surface areas $\left(13,000 \mathrm{~nm}^{2}\right)$ lay within this range of values expected for an aggregate nanoparticle structure. The discrepancy and the relatively low value of surface area measured by the HAADF-STEM tomography compared to the direct electrochemical measurement, is likely to be a consequence of the differing length scales at which these two measurements have been made. The HAADF-STEM tomography has a voxel resolution of the order of ca. $2 \mathrm{~nm}$ and hence able to evidence the presence of larger pores in the internal particle structure, while the electrochemical technique is ultimately a molecular level measurement. Although issues such as porous nanoparticle agglomeration may feasibly lead to higher measured surface area (see SI section 8 for details on the nanoparticle sol stability), the discrepancy between the surface areas measured by these two techniques most likely reflects the presence of smaller $1<2$ $\mathrm{nm}$ ) micropores or a high surface tortuosity which is unresolvable in the HAADF-STEM tomographic data. Full atomic resolution reconstructions are achievable for smaller nanoparticles ${ }^{26,33}$ but it is challenging to apply these techniques to the $\sim 30 \mathrm{~nm}$ diameter porous nanostructures considered in this work. An interesting area of future work would be to compare atomic resolution electron tomography data to single particle electrochemical measurements.

\section{Conclusions}

The volume of catalytically active material and its associated surface area, assessed on a per particle basis, are both crucial measurands for defining the chemical behaviour of nanoparticulate systems. However, these values can be challenging to obtain for complex aggregated nanostructures larger than approximately $10 \mathrm{~nm}$ diameter. This work has demonstrated that quantitative analysis of 2D HAADF-STEM images using a model nanoparticle standard can provide estimates for the density distribution of individual Pt nanoparticles, with the mean values found to be consistent with those obtained from 3D electron tomographic analysis. Furthermore, single nanoparticle electrochemistry 
measurements have been compared to electron tomographic surface area estimates. It is found that the electron tomography data gives a lower estimate of surface area, most likely as a result of spatial resolution limitations in the tomographic imaging of larger complex nanoporous structures.

\section{Acknowledgements}

SJH, SS and YW acknowledge funding from the European Research Council H2020 Starter Grant EvoluTEM, EPSRC (EP/P009050/1 and the NowNano CDT) and studentship funding from the China Scholarship Council.

\section{References}

1. H. Zheng, R. K. Smith, Y.-w. Jun, C. Kisielowski, U. Dahmen and A. P. Alivisatos, Science, 2009, 324, 1309-1312.

2. J. Park, H. Elmlund, P. Ercius, J. M. Yuk, D. T. Limmer, Q. Chen, K. Kim, S. H. Han, D. A. Weitz and A. Zettl, Science, 2015, 349, 290-295.

3. B. H. Kim, J. Yang, D. Lee, B. K. Choi, T. Hyeon and J. Park, Adv. Mater., 2018, 30, 1703316.

4. N. C. Bigall, T. Hartling, M. Klose, P. Simon, L. M. Eng and A. Eychmuller, Nano Lett., 2008, 8, 4588-4592.

5. Y. Sun and Y. Xia, Science, 2002, 298, 2176-2179.

6. A. Gole and C. J. Murphy, Chem. Mater., 2004, 16, 3633-3640.

7. $\quad$ N. G. Bastús, J. Comenge and V. Puntes, Langmuir, 2011, 27, 11098-11105.

8. N. G. Bastús, F. Merkoçi, J. Piella and V. Puntes, Chem. Mater., 2014, 26, 2836-2846.

9. N. Tian, Z.-Y. Zhou, S.-G. Sun, Y. Ding and Z. L. Wang, Science, 2007, 316, 732-735.

10. W. T. Nichols, T. Sasaki and N. Koshizaki, J. Appl. Phys., 2006, 100, 114911.

11. H. Plank, C. Gspan, M. Dienstleder, G. Kothleitner and F. Hofer, Nanotechnology, 2008, 19, 485302.

12. Y. Tu, F. Peng, X. Sui, Y. Men, P. B. White, J. C. M. van Hest and D. A. Wilson, Nature Chemistry, 2017, 9, 480.

13. Y. Li, K.-H. Yun, H. Lee, S.-H. Goh, Y.-G. Suh and Y. Choi, Biomaterials, 2019, 197, 12-19.

14. A. Samadi, H. Klingberg, L. Jauffred, A. Kjær, P. M. Bendix and L. B. Oddershede, Nanoscale, 2018, 10, 9097-9107.

15. Z. p. Xiang, H. q. Deng, P. Peljo, Z. y. Fu, S. I. Wang, D. Mandler, G. q. Sun and Z. x. Liang, Angew. Chem., 2018, 130, 3522-3526.

16. R. Dasari, D. A. Robinson and K. J. Stevenson, J. Am. Chem. Soc., 2013, 135, 570-573.

17. D. Dollimore, P. Spooner and A. Turner, Surface Technology, 1976, 4, 121-160.

18. Gemini V Operator's Manual, Micromeritics Instrument Corporation, 2006.

19. S. S. C. P. c. International Organization for Standardization. Technical Committee Iso/Tc 24. Particle characterization including sieving, Determination of the Specific Surface Area of Solids by Gas Adsorption: BET Method, ISO, 2010.

20. A. Kongkanand and M. F. Mathias, J. Phys. Chem. Lett., 2016, 7, 1127-1137.

21. K. Mayrhofer, Ph.D. Thesis, Vienna University of Technology, 2006.

22. X. Jiao, C. Batchelor-McAuley, N. P. Young and R. G. Compton, PCCP, 2018, 20, 23847-23850.

23. Z. Y. Li, J. Yuan, Y. Chen, R. E. Palmer and J. P. Wilcoxon, Appl. Phys. Lett., 2005, 87, 243103.

24. Z. Y. Li, J. Yuan, Y. Chen, R. E. Palmer and J. P. Wilcoxon, Adv. Mater., 2005, 17, 2885-2888.

25. P. M. Voyles, D. A. Muller, J. L. Grazul, P. H. Citrin and H. J. Gossmann, Nature, 2002, 416, 826. 
26. M. C. Scott, C.-C. Chen, M. Mecklenburg, C. Zhu, R. Xu, P. Ercius, U. Dahmen, B. C. Regan and J. Miao, Nature, 2012, 483, 444.

27. O. Ersen, I. Florea, C. Hirlimann and C. Pham-Huu, Mater. Today, 2015, 18, 395-408.

28. H. Rösner, S. Parida, D. Kramer, C. A. Volkert and J. Weissmüller, Adv. Eng. Mater., 2007, 9, 535-541.

29. C. T. Rueden, J. Schindelin, M. C. Hiner, B. E. DeZonia, A. E. Walter, E. T. Arena and K. W. Eliceiri, BMC bioinformatics, 2017, 18, 529.

30. B. D. A. Levin, Y. Jiang, E. Padgett, S. Waldon, C. Quammen, C. Harris, U. Ayachit, M. Hanwell, P. Ercius, D. A. Muller and R. Hovden, Microscopy Today, 2018, 26, 12-17.

31. B. Goris, W. Van den Broek, K. J. Batenburg, H. H. Mezerji and S. Bals, Ultramicroscopy, 2012, 113, 120-130.

32. Y.-C. Wang, T. J. A. Slater, G. M. Leteba, A. M. Roseman, C. P. Race, N. P. Young, A. I. Kirkland, C. I. Lang and S. J. Haigh, Nano Lett., 2019.

33. A. Varambhia, L. Jones, A. London, D. Ozkaya, P. D. Nellist and S. Lozano-Perez, Micron, 2018, 113, 69-82.

34. K. H. W. Van den Bos, L. Janssens, A. De Backer, P. D. Nellist and S. Van Aert, Ultramicroscopy, 2019, 203, 155-162.

35. R. Streubel, G. Bendt and B. Gökce, Nanotechnology, 2016, 27, 205602.

36. W. Van den Broek, A. Rosenauer, B. Goris, G. T. Martinez, S. Bals, S. Van Aert and D. Van Dyck, Ultramicroscopy, 2012, 116, 8-12.

37. Z. Zhong, R. Aveyard, B. Rieger, S. Bals, W. J. Palenstijn and K. J. Batenburg, Ultramicroscopy, 2018, 184, 57-65.

38. S. Trasatti and O. A. Petrii, Pure Appl. Chem., 1991, 63, 711-734.

39. K. J. J. Mayrhofer, D. Strmcnik, B. B. Blizanac, V. Stamenkovic, M. Arenz and N. M. Markovic, Electrochim. Acta, 2008, 53, 3181-3188. 